Bundesgesundheitsbl 2013 · 56:56-66 DOI 10.1007/s00103-012-1585-1

Online publiziert: 19. Dezember 2012

c) Springer-Verlag Berlin Heidelberg 2012
G. Krause · C. Frank · A. Gilsdorf · M. Mielke · L. Schaade · K. Stark · R. Burger Robert Koch-Institut, Berlin

\section{Der HUS-Ausbruch 2011 in Deutschland}

\section{Herausforderungen für den Infektionsschutz: Was sollte verbessert werden?}

Von Mai bis Juli 2012 ereignete sich in Norddeutschland der weltweit bisher größte Ausbruch des hämolytischurämischen Syndroms (HUS), der auch im Rückblick eine Bedrohung der öffentlichen Gesundheit war: Es handelte sich um schwere Krankheitsbilder mit relevanter Sterblichkeit auch bei Menschen ohne Vorerkrankungen; viele Patienten mussten intensivmedizinisch und mit Dialyse behandelt werden. Die Therapieoptionen waren begrenzt und mit unklarer Wirksamkeit. Die Fallzahlen stiegen innerhalb von Tagen steil an, ohne Gewissheit, wann der Ausbruch beendet sein würde. Das Ereignis beherrschte über Wochen die Medienberichterstattung im In- und Ausland und hatte neben den dramatischen Folgen für die Patienten auch enorme wirtschaftliche Folgen insbesondere im Bereich der Landwirtschaft. Allein die Entschädigungszahlungen der Europäischen Union an europäische Lebensmittelbetriebe betrugen 178 Mio. Euro [1].

Nachfolgend sollen besondere Aspekte der Bewältigung dieses Ausbruchs mit Schwerpunkt auf der Ausbrucherkennung und -erfassung, den epidemiologischen Untersuchungen, dem Ausbruchmanagement und der Kommunikation diskutiert und daraus folgende Empfehlungen zur Verbesserung von Verfahren und Strukturen erstellt werden.

Aspekte der Lebensmittelrückverfolgung, der internationalen Zusammenarbeit und der Patientenversorgung werden nur insofern berührt, als dies für die
Bewertung des Kernthemas erforderlich ist. Neben deutsch- und englischsprachigen Veröffentlichungen in medizinischen Fachzeitschriften, wurden Medienberichte sowie Kongressbeiträge und andere Berichte berücksichtigt, sofern sie im Internet zitiert sind. Zusätzlich wurden eigene, bislang unveröffentlichte deskriptive statistische und qualitative Auswertungen von Daten vorgenommen, die das RKI eigenständig erhoben oder aber im Rahmen des Infektionsschutzgesetzes (IfSG) übermittelt bekommen hat.

\section{Ergebnisse und Beobachtungen}

\section{Epidemiologische Untersuchung und Surveillance}

\section{Epidemiologische Untersuchung}

Im Rückblick wurde deutlich, dass der Ausbruch Anfang Mai 2011 begonnen und seinen Höhepunkt am 22.05 hatte (• Abb. 1). Am 26.07 wurde er für beendet erklärt [2]. Der letzte übermittelte, zum Ausbruch gehörige Erkrankungsbeginn war der 04.07. Hauptsächlich waren die Bundesländer Hamburg, Schleswig Holstein, Bremen, Mecklenburg-Vorpommern und Niedersachsen betroffen $[2,3,4,5]$. Im Ausland traten ebenfalls Fälle auf, die sich in einem dieser Bundesländer aufgehalten hatten. Insgesamt wurden in Deutschland 3793 Erkrankungen erfasst, davon hatten 827 Patienten ein HUS und 53 verstarben $[4,6]$. Bei Patienten mit Gastroenteritis lag der Altersmedian bei 46 Jahren, und 58\% wa- ren weiblich, bei Patienten mit HUS waren es im Median 43 Jahre und 69\% [2, 7] Frauen.

Die Inkubationszeit hatte einen $\mathrm{Me}$ dian von 8 Tagen [4]. Der überwiegende Teil der in Ausbruchclustern untersuchten Fälle ließ sich auf den Verzehr von Sprossen zurückführen. Nach den Erkenntnissen der Lebensmittelbehörden waren die Sprossen in einem niedersächsischen Betrieb produziert worden [8].

Das Robert Koch-Institut (RKI) hatte in enger Abfolge und zum Teil zeitgleich 13 epidemiologische Vor-Ort-Untersuchungen in Absprache mit den örtlichen Behörden durchgeführt (• Tab. 1). Hinzu kamen je eine Kohortenstudie der dänischen, schwedischen und niedersächsischen Behörden $[9,10,11]$. In all diesen Untersuchungen wurden - außer in den unter 4 und 5 in $\bullet$ Tab. 1 genannten Studien - die Patienten ausdrücklich nach Sprossenverzehr befragt. Erst in der vierten Fall-Kontroll-Studie und der Rezeptbasierten Kohortenstudie des RKI konnten signifikante Assoziationen zwischen Sprossenverzehr und Erkrankung nachgewiesen werden [8]. Nacherhebungen im Rahmen der vierten Fall-KontrollStudie hatten außerdem ergeben, dass 3 von 8 wieder befragten Fällen, die einen Sprossenverzehr zunächst verneint hatten, sich doch an diesen erinnerten, nachdem Sprossen durch die Ergebnisse der späteren Untersuchungen in den Medien diskutiert worden waren. Dagegen war dies bei keiner der 37 wieder befragten Kontrollen der Fall $[12,13]$. 


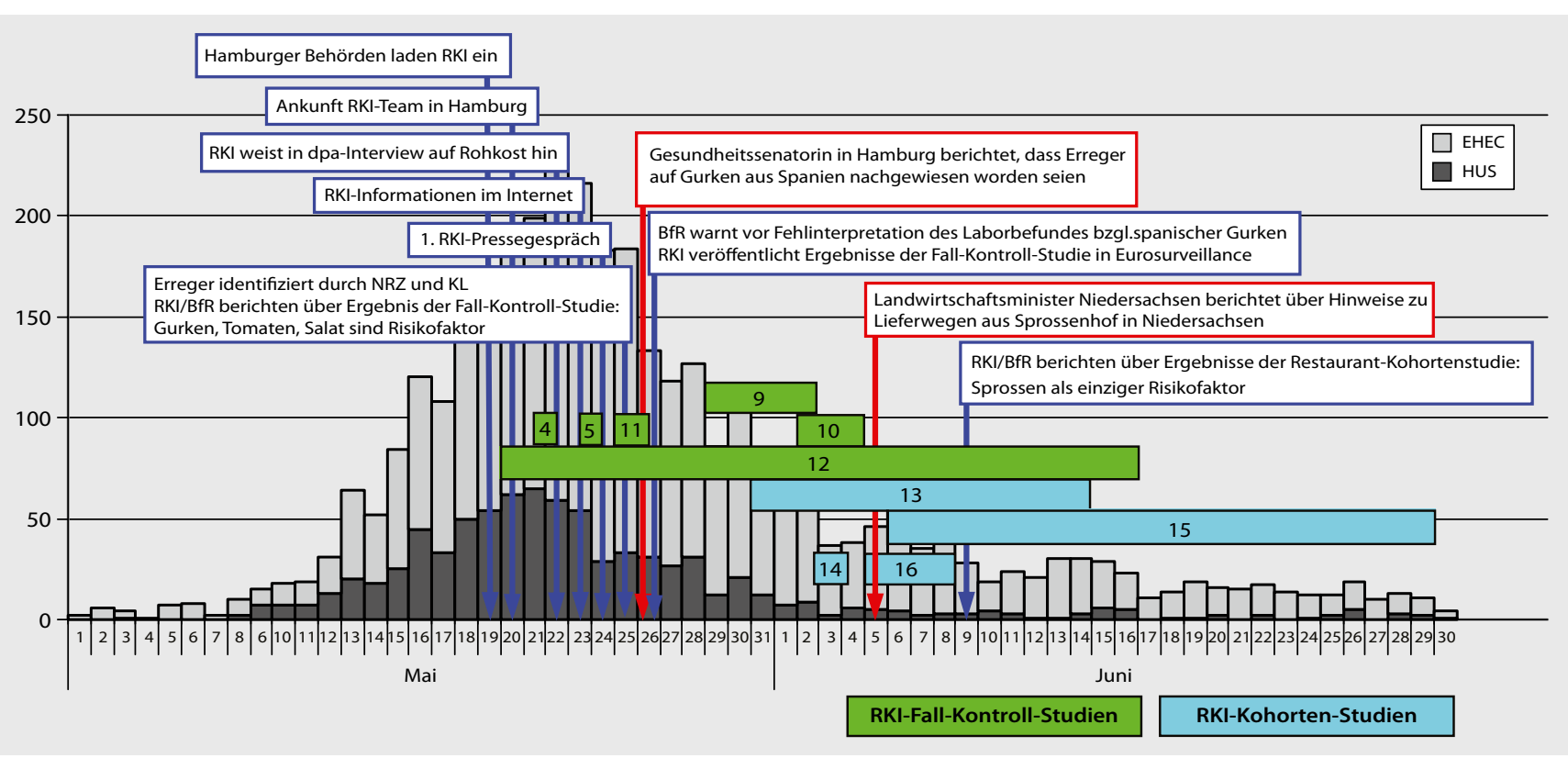

Abb. 1 A Epidemiologische Studien des RKI (Zahlen entsprechen den in Tab. 1 aufgeführten Studien), sowie wichtige behördliche Mitteilungen im zeitlichen Ablauf vor dem Hintergrund der epidemiologischen Kurve (Anzahl übermittelter Fälle nach Symptombeginn)

Am 05.06. hatten niedersächsische Behörden erste Hinweise veröffentlicht, dass Sprossenlieferungen eines Betriebes in Niedersachsen mit dem Ausbruch zusammenhingen [8]. Das übereinstimmende molekulare Erregerprofil und die Lieferwege von Sprossensamen im deutschen Ausbruch sowie in einem entsprechenden, epidemiologisch unabhängigen Ausbruch in Frankreich legen nahe, dass bereits die Sprossensamen kontaminiert gewesen sein müssen, bevor diese nach Deutschland bzw. Frankreich geliefert wurden $[14,15,16]$.

\section{Ausbrucherkennung}

Entdeckt wurde der Ausbruch durch eine Meldung an ein Gesundheitsamt in Hamburg am 18.05.2011. Es wurde eine Häufung von 3 HUS-Erkrankungen bei Kindern aus einem Krankenhaus gemeldet, woraufhin die Hamburger Gesundheitsbehörden am 19.05. das RKI um Unterstützung baten. Bei späteren retrospektiven Analysen wurde deutlich, dass bis zum 17.05. Ärzte und Labore in insgesamt 38 Landkreisen 51 Fälle als HUS bzw. EHEC diagnostiziert hatten, davon waren 8 allein im Einzugsgebiet eines Gesundheitsamtes, 3 Fälle im Einzugsgebiet eines weiteren Gesundheitsamtes und je 2 Fälle in den Einzugsgebieten von 2 weiteren
Gesundheitsämtern; die übrigen 36 Fälle traten in den Einzugsgebieten von 36 weiteren Gesundheitsämtern auf. Der ÖGD konnte diese Häufung jedoch bis dahin nicht erkennen, denn bis zum 18.05. hatte er nur 17 dieser 51 Fälle (33\%) als Meldung erfasst, und zwar nur jeweils eine Meldung pro Gesundheitsamt. Bis zum 15.5. gab es bereits 2 Land- bzw. Stadtkreise (keines von beiden war Hamburg), in denen je 2 bzw. 5 zum Ausbruch gehörige Fälle ärztlich diagnostiziert waren. Als Meldung gingen diese Diagnosen jedoch erst zwischen dem 23. und 29.05. bei den Gesundheitsämtern ein.

\section{Identifizierung und molekulare Charakterisierung des Erregers}

Nach Eingang der ersten diagnostischen Proben am 20.05. hatten das Nationale Referenzzentrum (NRZ) für Salmonellen und andere bakterielle Enteritiserreger am RKI und das RKI-Konsiliarlabor (KR) für HUS an der Universität Münster zeitgleich am 25.05. den Erreger als Shigatoxin 2 produzierenden E. coli 104:H4 identifiziert und weitere charakterisierende Eigenschaften wie Virulenzplasmide, den MLST-Sequenztyp, das Antibiogramm, das PFGE-Muster sowie detaillierte Anleitungen zum routinemäßigen Nachweis des Erregers in anderen Labo- ren veröffentlicht $[17,18,19,20]$. Der Ausbruchstamm wurde europäischen Referenzlaboren zur Verfügung gestellt.

\section{Erfassung der Versorgungslage}

Am 07.06. veröffentlichte das Universitätsklinikum Hamburg Eppendorf einen Aufruf zur Blutspende, weil zur Versorgung der HUS-Patienten innerhalb von 3 Wochen mehr Plasmakonzentrate verbraucht wurden als sonst innerhalb von 3 Monaten [21, 22]. Die Europäische Kommission stellte daraufhin mit Verweis auf genannten Aufruf die Frage, inwieweit die nephrologischen und intensivmedizinischen Versorgungsstrukturen in Deutschland in der Lage seien, die steigende Zahl an Patienten zu versorgen und ob Unterstützung aus dem europäischen Ausland organisiert werden solle. Daten zur Versorgungslage - z. B. der ggf. noch mobilisierbaren Dialyseplätze - lagen den Behörden während des eigentlichen Ereignisses nicht vor.

\section{Ausbruchmanagement}

\section{Mobilisierung personeller Ressourcen}

Allein für epidemiologische Untersuchungen, das Lagezentrum am RKI und die Beteiligung in der Task Force am Bun- 
desamt für Verbraucherschutz und Lebensmittelsicherheit (BVL) waren vonseiten des RKI zeitweise bis zu 70 wissenschaftliche Mitarbeiterinnen und Mitarbeiter und 15 Verwaltungskräfte gleichzeitig im Einsatz, von denen etwa 90\% aus Organisationseinheiten zusammengezogen wurden, die unter normalen Bedingungen für andere Funktionen oder Themen zuständig sind. Hinzu kamen über 10 Personen für die Laboraktivitäten und etwa 7 Personen für die Öffentlichkeitsarbeit, die ebenfalls teilweise aus anderen Bereichen mobilisiert wurden. Insgesamt waren am RKI über 110 Personen unmittelbar mit der Ausbruchaufklärung und -bewältigung befasst.

Vor Ort waren 13 Teams mit 2 bis 15 Personen im Einsatz. Diese konnten größtenteils Arbeitsräume in den Gesundheitsämtern und Krankenhäusern nutzen und wurden bei den eigentlichen Untersuchungen vereinzelt unterstützt durch Personal des örtlichen Gesundheitsamtes.

\section{Koordination}

Das RKI hatte im Rahmen seines Infektionsalarmplanes unmittelbar nach dem Hilfeersuchen der Hamburger Behörden ein Ausbruchuntersuchungsteam nach Hamburg geschickt und am Montag dem 23.05. das epidemiologische Lagezentrum aktiviert. Am 03.06. richtete das BVL die nationale Task Force EHEC in Berlin ein, an der Vertreter der betroffenen Bundesund Landesbehörden sowie der Europäischen Agentur für Lebensmittelsicherheit (EFSA) beteiligt waren. Ziel der Taskforce war, die vielfältigen Informationen zur Lebensmittelbeprobung und -rückverfolgung zusammenzutragen und zu bewerten [23, 24]. Am 23.05. hatte das RKI die erste der zunächst täglichen, später durchschnittlich zweitäglichen Telefonschaltkonferenzen (TSK) mit den Seuchenreferenten der Bundesländer einberufen. Hinzu kamen fast ebenso häufig TSK mit den Bundes- und Landesbehörden im Bereich der Lebensmittelsicherheit, mit Partnern der Europäischen Union sowie etwa zweimal wöchentlich mit der Weltgesundheitsorganisation (WHO). Zusätzlich organisierte das RKI insgesamt 6 TSK mit Vertretern der medizinischen Fachgesellschaften, betroffenen Kliniken und inter-

Bundesgesundheitsbl 2013 · 56:56-66 DOI 10.1007/s00103-012-1585-1

(c) Springer-Verlag Berlin Heidelberg 2012

\section{G. Krause · C. Frank · A. Gilsdorf · M. Mielke · L. Schaade · K. Stark · R. Burger Der HUS-Ausbruch 2011 in Deutschland. Herausforderungen für den Infektionsschutz: Was sollte verbessert werden?}

\section{Zusammenfassung}

Von Mai bis Juli 2012 ereignete sich in Norddeutschland der weltweit bisher größte Ausbruch von Erkrankungen am hämolytischurämischen Syndrom (HUS) mit schweren Folgen für die unmittelbar Betroffenen, das Gesundheitssystem und die Lebensmittelindustrie. Im Folgenden untersuchen wir im Bereich des Infektionsschutzes Aspekte der Ausbrucherkennung, des Ausbruchmanagements und der Kommunikation anhand wissenschaftlicher Publikationen, von Medienberichten und neuen eigenen Datenauswertungen. Die daraus folgenden 17 Empfehlungen betreffen unter anderem die konsequentere Beteiligung an bzw. Umsetzung bestehender und neuer Surveillancesysteme insbesondere vonseiten der Ärzteschaft, die breite Anwendung von Feintypisierungsdiagnostik, die personelle Verstärkung und effi- zientere Krisenmanagementstrukturen im öffentlichen Gesundheitsdienst (ÖGD) und die evidenzbasierte Kommunikation seitens der Behörden und wissenschaftlichen Fachgesellschaften. Ausbrüche ähnlicher Dimension können jederzeit wieder auftreten und immense Kosten verursachen, die entsprechende Investitionen in deren Früherkennung und Eindämmung bei Weitem übersteigen. Diese gesellschaftliche Gesamtbilanz sollte trotz knapper Ressourcen im öffentlichen Gesundheitsdienst entsprechend berücksichtigt werden.

\section{Schlüsselwörter}

Hämolytisch-urämisches Syndrom · E. coli · Ausbruch - Öffentlicher Gesundheitsdienst . Evaluation - Kommunikation - Management . Surveillance

\section{The 2011 HUS epidemic in Germany. Challenges for disease control: what should be improved?}

\section{Abstract}

From May to July 2012 the world's largest outbreak of hemolytic uremic syndrome (HUS) occurred in northern Germany with dramatic consequences for the population, the health care system and the food industry. In the following we examine the detection of the outbreak, epidemic management and related public communication aspects based on scientific publications, media reports as well as own and new data analyses. The subsequent 17 recommendations concern issues such as participation in and implementation of existing and new surveillance systems particularly with respect to physicians, broad application of finely tuned microbiological typ- ing, improved personnel capacity and crisis management structures within the public health service and evidence-based communication by administrations and scientific associations. Outbreaks of similar dimensions can inevitably occur again and result in costs which will far exceed investments needed for early detection and control. This societal balance should be taken into account in spite of limited resources in the public health sector.

\section{Keywords}

Hemolytic uremic syndrome - Outbreak .

E. coli . Public health service - Evaluation .

Communication $\cdot$ Management $\cdot$ Surveillance nationalen Experten, um aktuelle Daten zu bewerten und bevorstehende Maßnahmen zu beraten [2].

\section{Kommunikation}

Das RKI veröffentlichte ab 23.05. täglich eine epidemiologische Lageeinschätzung im Internet. Die Pressemitteilungen und Pressekonferenzen vom Bundesinstitut für Risikobewertung (BfR), BVL und RKI erfolgten überwiegend gemeinsam [25]. Pressemitteilungen und -kon- ferenzen der Landesgesundheitsministerien, Landesverbraucherschutzministerien oder deren nachgeordneten Institute fanden in der Regel einzeln statt. Hinzu kamen auf kommunaler Ebene Pressemitteilungen zu Todesfällen und Patientenzahlen in den jeweiligen Landkreisen oder Städten. Zusätzlich gab es offizielle Lageberichte des European Centre for Disease Prevention and Control (ECDC).

Inhaltlich sich unterscheidende behördliche Mitteilungen gab es insbesondere, als die Hamburger Gesundheitsse- 


\begin{tabular}{|c|c|c|c|c|c|c|}
\hline & $\begin{array}{l}\text { Erhebungszeit- } \\
\text { raum }\end{array}$ & Methodik & $\begin{array}{l}\text { Studienpopula- } \\
\text { tion }\end{array}$ & Ort & $\begin{array}{l}\text { Ergebnisse (statis- } \\
\text { tisch signifikant) }\end{array}$ & Quellen \\
\hline 1 & $20 / 05 / 2011$ & Exploration & 14 Fälle & Hamburg & $\begin{array}{l}\text { Fokussierung auf } \\
\text { pflanzliche Lebens- } \\
\text { mittel }\end{array}$ & [11] \\
\hline 2 & $29 / 05 / 2011$ & Exploration & 32 Fälle & Hamburg & $\begin{array}{l}\text { Fokussierung auf } \\
\text { pflanzliche Lebens- } \\
\text { mittel }\end{array}$ & [11] \\
\hline 3 & $02 / 06 / 2011$ & Exploration & 20 Fälle & $\begin{array}{l}\text { Lübeck, Bremer- } \\
\text { haven, Bremen }\end{array}$ & $\begin{array}{l}\text { Fokussierung auf } \\
\text { pflanzliche Lebens- } \\
\text { mittel }\end{array}$ & [11] \\
\hline 4 & $22 / 05 / 2011$ & Fall-Kontroll-Studie & $\begin{array}{l}14 \text { Fälle, } 16 \text { Kon- } \\
\text { trollen im Kranken- } \\
\text { haus }\end{array}$ & Hamburg & Tomaten, Gurken & [11] \\
\hline 5 & $24 / 05 / 2011$ & Fall-Kontroll-Studie & $\begin{array}{l}25 \text { Fälle, } 96 \text { Kon- } \\
\text { trollen in der Nach- } \\
\text { barschaft }\end{array}$ & Hamburg & $\begin{array}{l}\text { Gurken, Tomaten, } \\
\text { Blattsalat }\end{array}$ & [5] \\
\hline 6 & $\begin{array}{l}\text { 07/06/2011- } \\
10 / 06 / 2011\end{array}$ & Kohortenstudie & $\begin{array}{l}72 \text { Gäste einer Ge- } \\
\text { burtstagsfeier, } 7 \\
\text { erkrankt }\end{array}$ & Niedersachsen & $\begin{array}{l}\text { Keine signifikante } \\
\text { Assoziation }\end{array}$ & [11] \\
\hline 7 & $\begin{array}{l}\text { 24/05/2011- } \\
31 / 05 / 2011\end{array}$ & Kohortenstudie & & $\begin{array}{l}\text { Schwedische Rei- } \\
\text { serückkehrer aus } \\
\text { Norddeutschland }\end{array}$ & $\begin{array}{l}\text { Keine signifikante } \\
\text { Assoziation }\end{array}$ & [10] \\
\hline 8 & $\begin{array}{l}30 / 05 / 2011- \\
02 / 06 / 2011\end{array}$ & Kohortenstudie & & $\begin{array}{l}\text { Dänische Reise- } \\
\text { rückkehrer aus } \\
\text { Norddeutschland }\end{array}$ & $\begin{array}{l}\text { Keine signifikante } \\
\text { Assoziation }\end{array}$ & [9] \\
\hline 9 & $\begin{array}{l}\text { 29/05/2011- } \\
02 / 06 / 2011\end{array}$ & Fall-Kontroll-Studie & $\begin{array}{l}46 \text { Fälle, } 2100 \text { Kon- } \\
\text { trollen im „online } \\
\text { panel“ }^{\prime \prime}\end{array}$ & $\begin{array}{l}\text { Lübeck, Hamburg, } \\
\text { Bremen }\end{array}$ & Blattsalat & [11] \\
\hline 10 & $\begin{array}{l}02 / 06 / 2011- \\
04 / 06 / 2011\end{array}$ & Fall-Kontroll-Studie & $\begin{array}{l}26 \text { Fälle, } 81 \text { Kon- } \\
\text { trollen in Nachbar- } \\
\text { schaft }\end{array}$ & $\begin{array}{l}\text { Lübeck, Bremer- } \\
\text { haven, Bremen }\end{array}$ & $\begin{array}{l}\text { Sprossen, Gurke, } \\
\text { Lachs, Ziegenkäse }\end{array}$ & [8] \\
\hline 11 & $26 / 05 / 2011$ & Fall-Kontroll-Studie & $\begin{array}{l}23 \text { Fälle, } 30 \text { Kont- } \\
\text { rollen in Firmen- } \\
\text { belegschaft }\end{array}$ & Frankfurt & Salat-Bar-Einkäufe & [40] \\
\hline 12 & $\begin{array}{l}\text { 20/05/2011- } \\
17 / 06 / 2011\end{array}$ & Fall-Kontroll-Studie & $\begin{array}{l}60 \text { Fälle, } 465 \text { Kon- } \\
\text { trollen Allgemein- } \\
\text { bevölkerung }\end{array}$ & $\begin{array}{l}\text { Lübeck, Bremer- } \\
\text { haven, Bremen, } \\
\text { Hamburg }\end{array}$ & $\begin{array}{l}\text { Sprossen, Salat, } \\
\text { Äpfel }\end{array}$ & [11] \\
\hline 13 & $\begin{array}{l}31 / 05 / 2011- \\
14 / 06 / 2011\end{array}$ & Kohortenstudie & $\begin{array}{l}\text { Reisegruppe: } \\
\text { 38 Teilnehmer, } 9 \\
\text { erkrankt }\end{array}$ & Deutschlandweit & Keine & [11] \\
\hline 14 & $03 / 062011$ & Kohortenstudie & $\begin{array}{l}22 \text { Mitarbeiter } \\
\text { einer Firma, } 6 \text { er- } \\
\text { krankt }\end{array}$ & Hamburg & Keine & [11] \\
\hline 15 & $\begin{array}{l}07 / 06 / 2011- \\
08 / 07 / 2011\end{array}$ & Kohortenstudie & $\begin{array}{l}\text { Reisegruppe: } \\
\text { 116 Teilnehmer, } 12 \\
\text { erkrankt }\end{array}$ & Deutschlandweit & $\begin{array}{l}\text { Überwiegend Roh- } \\
\text { kost in verschie- } \\
\text { denen Mahlzeiten } \\
\text { (z. B. Salat, Toma- } \\
\text { ten, Gurken) }\end{array}$ & [11] \\
\hline 16 & $\begin{array}{l}\text { 06/06/2011- } \\
09 / 06 / 2011\end{array}$ & Kohortenstudie & $\begin{array}{l}\text { Verschiedene } \\
\text { Gruppen: } 168 \text { Teil- } \\
\text { nehmer, } 31 \text { er- } \\
\text { krankt }\end{array}$ & Lübeck & Sprossen & [8] \\
\hline
\end{tabular}

natorin in einer Pressekonferenz mitteilte, ein EHEC-Erreger sei auf spanischen Gurken nachgewiesen worden, woraufhin das BfR noch am selben Tag vor Fehlinterpretationen dieses Befundes warnte [26].
Auch Fachgesellschaften und medizinische Berufsverbände hatten sich aktiv an der Kommunikation beteiligt, indem sie entweder die aktuellen Ereignisse und Maßnahmen bewerteten oder aber Empfehlungen aussprachen. Hierzu gehörten Fachempfehlungen der Deutschen Gesellschaft für Infektiologie und der Deutschen Gesellschaft für Nephrologie zu Fragen der Therapie, auf die das 
RKI im Rahmen seiner Veröffentlichungen verwiesen hat [27, 28]. Zugleich kritisierten die Deutsche Gesellschaft für Allgemeinmedizin und die deutsche Gesellschaft für Krankenhaushygiene bzw. ihre Vertreter die Empfehlungen von RKI und BfR, ohne die jeweils verfügbaren Ermittlungsergebnisse zu berücksichtigen [29, 30, 31]. Hinzu kamen zahlreiche Äußerungen von Wissenschaftlern, in denen sich diese zum Teil selbst oder dem jeweiligen Sachstand widersprachen [32, 33, 34, 35]. So hatten z. B. 2 Lehrstuhlinhaber für Mikrobiologie in Presseinterviews zu einem Zeitpunkt Fleischprodukte als mögliche Quelle für diesen Ausbruch hervorgehoben, als Fleisch durch die bereits veröffentlichten epidemiologischen Untersuchungen des RKI als Infektionsvehikel schon weitgehend ausgeschlossen werden konnte [36, 37].

\section{Diskussion}

Während des Ausbruchs und im Nachgang wurde die öffentliche Diskussion geprägt von Fragen, ob er von den Gesundheitsbehörden rechtzeitig erkannt und berichtet worden ist, ob er sachgerecht untersucht wurde und ob die Kommunikation von offizieller Seite sachgerecht verlief. Die oben aufgeführten Informationen und Auswertungen machen deutlich, dass die Antworten auf diese Fragen bisher wenig beachtete Aspekte hervorbringen, aus denen konkrete Empfehlungen ableitbar sind:

\section{Epidemiologische Untersuchung und Surveillance}

\section{Epidemiologische Untersuchungen}

Obwohl in den ersten Erhebungen direkt nach Sprossenverzehr gefragt worden war, konnte dieser erst nach einiger Verzögerung in einer Fall-Kontroll-Studie und einer Kohortenstudie mit dem Ausbruchgeschehen in Verbindung gebracht werden. Dies erklärt sich zum einen dadurch, dass sich viele Patienten insbesondere anfänglich nicht erinnerten, Sprossen verzehrt zu haben, weil sie diese nicht selbst zubereitet oder gekauft hatten und häufig nur in kleinen Mengen und unbewusst als Garnierung oder Teil einer
Salatbeilage verzehrt hatten. Zum anderen konnte erst durch die epidemiologischen Untersuchungen in der ersten Woche festgestellt werden, dass die Inkubationszeit bei diesem Stamm zwischen 2 und 15 Tagen lag und damit deutlich länger war als die 2 bis 4 Tage, die bislang für EHEC bekannt waren. Die ersten Befragungen bezogen sich jedoch auf die letzte Woche vor Erkrankungsbeginn. Naturgemäß fiel es den Befragten schwerer, sich daran zu erinnern, welche Lebensmittel sie in einem Zeitraum von 2 Wochen vor Erkrankungsbeginn verzehrt hatten. Da nur 25\% der Patienten anfänglich Sprossenverzehr angegeben hatten [13], war es im Einklang mit bestehenden WHOEmpfehlungen [38], den Sprossenverzehr in den ersten beiden Fall-KontrollStudien nicht zu erheben. Die Erkenntnis aus den Wiederbefragungen im Rahmen der vierten Fall-Kontroll-Studie sowie die ergebnislosen 6 Kohortenstudien im In- und Ausland zeigen aber, dass hier keinesfalls ein methodisches Versäumnis in der Fragebogengestaltung vorlag [35], sondern vielmehr die Expositionserfassung auf Grundlage des Erinnerungsvermögens nicht sensitiv genug war und zum Teil auch die Dokumentation der angebotenen Speisen durch die Gastronomiebetriebe hierfür nicht ausreichend war.

Ähnliche Erfahrungen wurden auch im Jahr 2008 in den USA bei einem Ausbruch mit insgesamt 1500 SalmonellaSaintpaul-Infizierten gemacht, bei dem die Erkrankungen zunächst mit Tomatenverzehr assoziiert wurden und erst im weiteren Verlauf die häufig in Tomatenzubereitungen verarbeiteten Chilischoten als Quelle identifiziert werden konnten [39]. Dieser Ausbruch wurde etwa 4 Wochen nach Auftreten der ersten Fälle als solcher erkannt und nach etwa 7 weiteren Wochen aufgeklärt. Dies ist nur ein Beispiel dafür, dass Lebensmittel, die kaum bewusst verzehrt werden, in Befragungen allein nur schwer erfasst werden können und daher die Auswertung von Bestell-, Abrechnungs- und Herstellungsdaten häufiger zum Einsatz kommen sollte [13, 16], so wie dies bei diesem Ausbruch in 2 Studien erfolgte $[8,40]$.

Die ersten Befragungen konnten frühzeitig und zutreffend ausschließen, dass Fleisch- oder Milchprodukte von Bedeu- tung für den Ausbruch sein könnten, sodass Lebensmittelüberwachungsämter das Spektrum der zu untersuchenden Lebensmittel rasch eingrenzen konnten. Allerdings lagen keine ausreichend detaillierten Daten über Verzehrgewohnheiten in der deutschen Bevölkerung vor, um bereits in dieser Phase spezifische Rohkostlebensmittel eingrenzen zu können.

Erst als für das RKI entsprechende Patientenkohorten durch die übermittelten Meldedaten bekannt wurden, konnten rezeptbasierte Studienansätze verfolgt werden.

\section{Ausbrucherkennung}

In Bezug auf die 3 HUS-Patienten, die in Hamburg Auslöser für das Erkennen des Ausbruchs waren, ist sowohl seitens der behandelnden Pädiater als des Gesundheitsamtes keinerlei Zeit versäumt worden. Aber die Auswertungen zeigen auch, dass innerhalb und außerhalb Hamburgs schon vor dem 18.05. Ärzte gehäuft EHEC- oder blutige Durchfallerkrankungen diagnostiziert, diese aber nur zum kleinen Teil an die Gesundheitsämter gemeldet hatten [41].

Die Diskussion, dass für die Übermittlung von Meldungen über die Landesbehörde an das RKI bislang bis zu 2 Wochen vorgesehen waren [24, 42, 43], berücksichtigt nicht, dass der Ausbruch vor allem durch eine bessere und raschere Diagnostik und Meldung durch die behandelnden Ärzte und Labore früher hätte entdeckt werden können. Auch wenn die dem RKI übermittelten Diagnosedaten im Einzelfall nicht immer präzise sind, so legen die oben präsentierten Auswertungen nahe, dass der Ausbruch 3 bis 4 Tage früher hätte erkannt werden können, wenn diese Diagnosen stets unverzüglich an das jeweilige Gesundheitsamt gemeldet worden wären. Insbesondere in der Ärzteschaft in der stationären und ambulanten Patientenversorgung scheint nicht allen ihre in der Früherkennung von Ausbrüchen so wichtige und gesetzlich geregelte Rolle ausreichend bewusst zu sein [44]. Als Folge der EHEC-Erfahrungen hat der Bundestag im Herbst 2011 eine Gesetzesänderung beschlossen, die nicht nur die Datenübermittlungszeiten vom Gesundheitsamt über die Landesbehörde zum RKI verkürzen würde, son- 
dern auch die Entwicklung eines deutschlandweiten Erhebungssystems vorsieht, das eine halbautomatische digitale Meldung bereits auf der Ebene der ärztlichen Patienten- und Labordokumentation an das Gesundheitsamt ermöglichen würde. Dieser Gesetzentwurf befindet sich jedoch zum Zeitpunkt der Drucklegung noch im Vermittlungsausschuss des Bundesrates und Bundestages [24].

Möglicherweise ist es auch kein Zufall, dass der Ausbruch zunächst bei Kindern entdeckt wurde, obwohl er überwiegend Erwachsene betraf, denn das RKI koordiniert - unterstützt von der Gesellschaft für Pädiatrische Nephrologie - seit Jahren eine freiwillige Erfassung von HUS bei 45 Kinderdialysezentren in Deutschland in Ergänzung zur gesetzlichen Meldepflicht [45]. Gerade dann, wenn genauere klinische oder labordiagnostische Erhebungen erforderlich sind, können Sentinels - also Erhebungssysteme, an denen eine Auswahl von Laboren oder Ärzten beteiligt sind - die Limitationen einer flächendeckenden Meldepflicht kompensieren. Der Bedarf nach Sentinelsystemen besteht nicht nur für Gastroenteritiden, sondern auch für eine Vielzahl anderer Erreger, und zwar vor allem dann, wenn eine gesetzliche Meldepflicht nicht besteht oder die benötigte Informationstiefe nicht bieten kann. Es fällt allerdings zunehmend schwerer, Labore, Krankenhäuser oder Arztpraxen zur freiwilligen und dauerhaften Beteiligung an solchen Erhebungssystemen zu motivieren [46, 47, 48]. Für einen finanziellen Ausgleich derartiger Aktivitäten der teilnehmenden Ärzte fehlen öffentliche Mittel.

Schwierigkeiten gab es auch bei der Erfassung von Todesfällen. Das RKI war vielfach darauf angewiesen, diese Informationen aus Pressemitteilungen betroffener Kommunen oder Krankenhäuser selbst zusammenzutragen, statt sich hier auf eine verlässliche Informationsübermittlung verlassen zu können. Es wird vielfach unterschätzt, dass auch die EU-Kommission, die WHO und das ECDC, legitimiert durch EU-Verordnungen und die internationalen Gesundheitsvorschriften, inländische Pressemeldungen auswerten und bei nationalen Behörden eine Bestätigung und Bewertung einfordern.

\section{Identifizierung und molekulare Charakterisierung des Erregers}

In Deutschland basiert die HUS-Diagnostik auf dem Nachweis der Shigatoxinbildung des verursachenden pathogenen E. coli. Eine Anzüchtung unterbleibt zumeist. Nach Eingang relevanter Proben beim NRZ bzw. dem KL gelang die Identifizierung eines neuartigen Erregers sehr rasch. Hilfreich war, dass der verantwortliche Erreger durch Nachweis der Extended-Spektrum- $\beta$-Lactamase (ESBL) in Kombination mit dem Shigatoxingen 2 ausreichend genau identifizierbar war. So konnten auch periphere Labore ohne Feintypisierungskapazität für E. coli den Ausbruchstamm nachweisen. Allerdings hätte der Ausbruch besser erfasst und möglicherweise auch früher erkannt werden können, wenn ein System zur Feintypisierung flächendeckend existiert hätte [13]. Als Standardbeispiel weltweit gilt hier das PulseNet, ein Labornetzwerk, das seit Jahren in den USA erfolgreich genutzt wird, immerhin einem ebenfalls großen, föderal strukturierten Land mit hochgradig privatisiertem Krankenversorgungssystem [49]. Es sollte daher erwogen werden, dem RKI in ähnlichen, eng zu definierenden Situationen eine rechtliche Grundlage zur direkten Informationserhebung und -übermittlung zu geben. Beispiel hierfür könnten das österreichische Epidemiegesetz mit seiner Änderung im Jahr 2008 oder das vom Schweizerischen Parlament 2012 verabschiedete Epidemiegesetz sein, in denen den jeweiligen Bundeseinrichtungen entsprechende Befugnisse zugesprochen werden [50, 56, 57]. Die Vereinigten Staaten von Amerika haben eine noch etwas weiter reichende, wenngleich kaum angewandte gesetzliche Regelung mit der Bezeichnung „measures in the event of inadequate local control“, die der zuständigen Bundesagentur im Bedarfsfall zusätzliche Befugnisse einräumt [61].

\section{Erfassung der Versorgungslage}

Der Aufruf zu vermehrten Blutspenden sowie die Entsendung von Dialysegeräten und Fachpersonal von weniger belasteten Krankenhäusern an solche mit entsprechendem Bedarf deutet an, dass einerseits die regulären Versorgungsstrukturen an die Grenzen geraten waren, andererseits die Mobilisierung der Versorgungskapazität im Rahmen der Selbstorganisation jedoch in diesem Fall gelang. Wie bereits während der Influenzapandemie 2009 wurde jedoch auch dieses Mal deutlich, dass in Deutschland kein Informationssystem verfügbar ist, das eine systematische Übersicht über die Versorgungslage der Patienten ermöglicht [51]. Das RKI sah sich im Verlauf des Ausbruchs gerade infolge des in Hamburg veröffentlichten Blutspendeaufrufs mit Anfragen der EU-Kommission konfrontiert, wie denn die Versorgungslage insbesondere in Bezug auf Dialyseplätze sei und ob hier Unterstützung durch andere Mitgliedstaaten erforderlich werden könnte. Es erscheint fraglich, ob die erforderliche Versorgungskapazität auch hätte aufrechterhalten werden können, wenn die ursächlichen Sprossen nicht nur in Norddeutschland, sondern bundesweit vertrieben und verzehrt worden wären oder wenn die Identifizierung der Ausbruchsursache bei fortwährender Exposition nicht 3 Wochen, sondern 7 Wochen gedauert hätte, wie dies beim oben erwähnten Ausbruch von Salmonella Saintpaul in den USA im Jahr 2008 der Fall war [39]. Nicht nur, um durch internationale Verträge legitimierte Anfragen beantworten zu können, sondern auch aus Fürsorgepflicht gegenüber der eigenen Bevölkerung sollte ein Staat in der Lage sein, zeitnah und flexibel die Versorgungslage $\mathrm{zu}$ überwachen. Dies ist notwendig, um entweder bevorstehende Kapazitätsengpässe zu erkennen oder aber um mit Bezug auf belastbare Daten der Öffentlichkeit versichern zu können, dass entsprechende Kapazitätsgrenzen nicht zu erwarten sind.

\section{Epidemiemanagement}

\section{Mobilisierung personeller Ressourcen}

Gesundheitsämter und Landesbehörden zeigten, vor allem nachdem die Dimension des Ausbruchs erkannt war, selten Vorbehalte, das RKI um Unterstützung zu bitten. Beim erwähnten SalmonellaSaintpaul-Ausbruch wurde die Bewältigung teilweise dadurch verzögert, dass örtliche Behörden in den USA gezögert hatten, Unterstützung nationaler Behörden anzufordern [52]. Auch beim vorlie- 
genden HUS-Ausbruch hat dies vereinzelt eine Rolle gespielt. Die Hamburger Behörden hatten das RKI allerdings unverzüglich eingebunden.

Im weiteren Verlauf sah sich das RKI mit deutlich mehr Unterstützungsersuchen konfrontiert, als es trotz Mobilisierung aller Ressourcen hätte leisten können. Wiederum ähnlich zum SalmonellaSaintpaul-Ausbruch [52] stießen die RKITeams vor Ort auf nur sehr begrenzte personelle Kapazität bei den örtlichen Behörden. Insgesamt erscheint die personelle Ausstattung des kommunalen öffentlichen Gesundheitsdienstes kaum ausreichend, um intensivere Ausbruchuntersuchungen eigenständig durchzuführen. Obgleich die Arbeitsbedingungen prinzipiell attraktiv erscheinen, fällt es offenkundig schwer, Personal für den ÖGD zu rekrutieren, möglicherweise auch weil die Einkommen, die dort erzielt werden, deutlich niedriger sind als in Krankenhäusern [53].

Infektionsschutz ist zudem nur eines von vielen Themen, mit denen sich das Personal in Gesundheitsämtern befassen muss. Qualifizierungsangebote hier$\mathrm{zu}$ werden unter anderem von Landesbehörden und dem RKI vorgehalten, stoßen aber hier auf Kapazitätsgrenzen, da die Fortbildung nicht zu den klassischen Aufgaben von Gesundheitsbehörden gehört [54].

Nur ausnahmsweise war erkennbar, dass auf örtlicher Ebene Kräfte aus anderen Behördenbereichen zusammengezogen wurden. Während Fachpersonal zur Dialysebehandlung von nicht betroffenen Krankenhäusern in betroffene Krankenhäuser verlegt wurde, sind derartige $\mathrm{Ab}$ ordnungen von betroffenen an weniger betroffene Gesundheitsämter nicht bekannt. Aus Sicht des RKI sollte der örtliche ÖGD eine qualifizierte epidemiologische Expertise besitzen, denn dort liegt die primäre Zuständigkeit für das Erkennen, Untersuchen und Eindämmen von Epidemien. Da derart spezialisierte Kräfte auf absehbare Zeit kaum in jedem Gesundheitsamt vorgehalten werden können, sollten zumindest auf Ebene der Landesbehörden infektionsepidemiologische Experten zur Unterstützung der örtlichen Behörden verfügbar sein. Da komplexe Ausbrüche pro Gesundheits- amt selten auftreten, wären hier überregionale, aber niedrigschwellig anforderbare Teams mit einem Fokus auf der epidemiologischen Untersuchung und Aufklärung von Krankheitsausbrüchen von Vorteil.

Im Vergleich zur Bevölkerung und zum Bruttoinlandsprodukt sind die USamerikanischen Gesundheitsbehörden auf der Ebene der föderalen Staaten personell deutlich besser ausgestattet als die Landesgesundheitsbehörden in Deutschland [55]. Entsprechendes gilt für die jeweiligen Bundesbehörden. Um, bezogen auf die Bevölkerungsgröße und beteiligten Behörden, ein Minimum an Unterstützung für die Gesundheitsämter leisten zu können, wären bei den Landebehörden mindestens 25 bis 30 zusätzliche infektionsepidemiologische Experten notwendig [54] - was allerdings nur dann ausreichen würde, wenn diese im kollegialen Austausch auch über die Bundeslandgrenzen hinaus einsetzbar wären.

\section{Koordination}

Aufgrund der dramatischen Umstände dieses Ausbruchs waren sehr intensive Untersuchungen und rasche Entscheidungen auf Basis der jeweils verfügbaren Erkenntnisse erforderlich. In anderen Bereichen - wie z. B. beim Katastrophenschutz oder bei Großunfällen - kommen in derartigen Situationen zumindest auf Landesebene zentralisierte Krisenmanagementverfahren zum Einsatz, um die Informationen und Aktivitäten aller beteiligten Akteure zu koordinieren. Hierfür wird eine technisch aufwendige und fortlaufend beübte Infrastruktur vorgehalten. Ein ressortübergreifendes Krisenmanagement erfordert jedoch nicht nur das Zusammenbringen der beteiligten Behörden. Für Ereignisse dieser Art wird eine moderne Management- und Informationsstruktur in räumlicher Nähe zu den betroffenen Behörden benötigt. Für den organisatorischen Ablauf bedarf es hierfür speziell geschulten, technischen Personals. Solch eine Struktur könnte beispielsweise vom Bundesamt für Bevölkerungsschutz und Katastrophenhilfe vorgehalten werden, allerdings müsste der Standort in Berlin sein, um eine zeitnahe Besetzung mit Experten aus den betroffenen Bundesbehörden und kurze Infor- mationswege zu ermöglichen. Der Vorteil einer solchen ressortunabhängigen Ansiedlung wäre außerdem, dass das Krisenmanagement an einem Ort verbliebe und lediglich die personelle Besetzung an den Bedarf angepasst würde. Wäre im besagten Ausbruch, nachdem die Lebensmittelaspekte weitgehend gelöst waren, der Erreger relevant von Mensch zu Mensch oder über die Umwelt verbreitet worden - wie dies zeitweise befürchtet wurde -, hätte die Beheimatung der Task Force auf das RKI oder das Umweltbundesamt wechseln müssen. Neben der technischen Infrastruktur muss auch die Federführung und Weisungskompetenz für solch ein Ereignis klar geregelt werden.

Die Zuständigkeiten der verschiedenen Ressorts und Behörden auf kommunaler, Landes- und Bundesebene wurden aus Sicht des RKI weitgehend respektiert. Allerdings waren durchaus Effizienzverluste zu beklagen. So darf das RKI außerhalb eines Forschungsprojektes keine personenbezogenen Daten erheben und verarbeiten und muss sämtliche Erhebungsbögen für nationale ergänzende Surveillancemaßnahmen mit den 16 Landesbehörden im Detail abstimmen. Das RKI kann nur auf Einladung örtlicher Behörden vor Ort aktiv werden und muss faktisch seine Aktivitäten immer mit ihnen abstimmen. In den meisten Situationen stellt dies kein Problem dar und ist auch aus fachlichen Gründen erwünscht. Bei diesem Ausbruch führte der Abstimmungsbedarf aber durchaus zu einem personellen Mehraufwand und zu Verzögerungen. Auch die Tatsache, dass Informationen über ein besonders betroffenes Restaurant nicht zeitgleich an das örtliche Veterinäramt und alle anderen zuständigen Behörden geschickt werden durften, sondern sequenziell über mehrere Verwaltungsebenen verschiedener Ressorts und Bundesländer laufen mussten, führte zu Verzögerungen. Tatsächlich sah sich das RKI vereinzelt gezwungen, jenseits seiner Zuständigkeit Informationen direkt an örtliche Behörden zu übermitteln, um wichtige Interventionen nicht zu verzögern. Es sollte daher erwogen werden, dem RKI in ähnlichen, eng zu definierenden Situationen eine rechtliche Grundlage zur direkten Informa- 
tionserhebung und -übermittlung zu geben. Beispiel hierfür könnte das österreichische Epidemiegesetz mit seiner Änderung im Jahr 2008 sein, in dem das Bundesministerium entsprechende Befugnisse erhält [50].

\section{Kommunikation}

\section{Abgestimmte Kommunikation}

Wenngleich es wünschenswert gewesen wäre, schon ganz zu Anfang die Sprossen als Ursache für den Ausbruch bekannt geben zu können, so war es auch in der Nachschau richtig, bereits die ersten epidemiologischen Ergebnisse, die auf Gurken, Tomaten und Blattsalat als Verursacher hinwiesen, mit dem Verweis auf die provisorische Datenlage zu kommunizieren. Ausländische Kommunikationsexperten erkannten an, dass die Bundesbehörden die unsichere Datenlage der ersten Empfehlungen von Beginn an offen, jedoch nicht offensiv, kommuniziert hatten. Die Kommunikation der Unsicherheit schien wiederum von manchen als Zeichen der Unfähigkeit interpretiert zu werden [26]. Divergierende behördliche Mitteilungen gab es, als einzelne Kommunen Erkrankungen, Folgeausbrüche und Todesfälle öffentlich bekannt machten, ohne das RKI zuvor darüber zu informieren. Ähnliche Abstimmungsprobleme gab es bei der Mitteilung über in Gewässern festgestellte bzw. vermeintlich festgestellte Erregernachweise. Besonders folgenschwer war jedoch die Warnung der Hamburger Gesundheitssenatorin vor spanischen Gurken, ohne die anderen betroffenen Behörden vorab über diese Absicht $\mathrm{zu}$ informieren und den Befund gemeinsam mit den fachkundigen Stellen zu bewerten $[26,59]$. Die Warnung des BfR vor einer Fehlinterpretation des Befundes fand in der Mediendynamik nicht das nötige Gehör [26, 60, 59]. Tatsächlich gibt es zwar im Rahmen des IfSG eine Verwaltungsvorschrift, die bestimmt, dass Bundesbehörden die Landesbehörden über entsprechende Mitteilungen vorab informieren; umgekehrt ist dies aber dort nicht vorgesehen.

Neben diesen potenziellen Reibungsverlusten und tatsächlichen Kommunikationslücken zwischen den Behörden unterschiedlicher föderaler Ebenen wurde die öffentliche Diskussion auch davon geprägt, dass in der Öffentlichkeit, aber auch bei klinisch tätigen Ärzten keine rechte Vorstellung darüber besteht, mit welchen Methoden, Möglichkeiten und Beschränkungen Infektionsausbrüche untersucht und eingedämmt werden müssen. Das gilt auch für die Medien, die in solchen Ausbruchgeschehen eine Schlüsselrolle einnehmen [61]. In der akuten Situation waren die betroffenen Behörden damit überfordert, diese Art der Aufklärungsarbeit zu leisten, zum einen, weil die hierfür erforderlichen Kapazitäten durch die Ausbruchuntersuchung gebunden waren, und zum anderen, weil während der laufenden Ermittlungen keine unzureichend gesicherten Befunde kommuniziert werden sollten. Das RKI z. B. machte zwar den täglichen Stand der Erkenntnisse öffentlich verfügbar, erläuterte aber nicht mit der gleichen Intensität die aktuellen und geplanten Aktivitäten. Dieses unbefriedigte Informationsbedürfnis hat offenkundig zu Spekulationen und unrealistischen Erwartungen geführt, die sich in entsprechenden Vorwürfen äußerten.

\section{Wissenschaftlicher Diskurs}

Die fachliche und kommunikative Unterstützung durch einige Fachgesellschaften und Experten in Telefonkonferenzen oder ad hoc erstellte wissenschaftliche Empfehlungen waren ein wichtiger Beitrag zur Bewältigung des Ausbruchs.

Zugleich trugen aber die oben nur beispielhaft zitierten, irreführenden Expertenkommentare und Mitteilungen von Berufs- und Fachgesellschaften vermutlich erheblich zum Vertrauensverlust gegenüber den behördlichen Empfehlungen bei. Dabei war das Informationsangebot des RKI im Internet zu jedem Zeitpunkt durchaus umfangreich genug und auch auf der Startseite gut sichtbar platziert, sodass selbst Kommentatoren, die nicht in die Ermittlungen eingebunden waren, in der Lage gewesen wären, unzutreffende Behauptungen zu vermeiden.

Es stellt sich die Frage, ob Behörden in der Lage sein müssen, derart offenkundige Fehlinformationen jeweils spezifisch richtigzustellen. Zweifellos muss auch in einer Krisensituation eine kritische Meinungsvielfalt gefördert werden, aber diese geht auch mit einer Verantwortung einher, die insbesondere wissenschaftliche Fachgesellschaften von ihren hoch qualifizierten, medienerfahrenen Vertretern einfordern müssen. Soweit erkennbar, fehlen in den derzeit bestehenden Empfehlungen zur guten wissenschaftlichen Praxis, die sich deutsche Fachgesellschaften in Bezug auf ethisch und fachlich korrektes Handeln gegeben haben, klare Hinweise, was beim Umgang mit öffentlichen Medien zu beachten ist.

\section{Empfehlungen}

\section{Epidemiologische Untersuchung und Surveillance}

\section{Epidemiologische Untersuchung}

1. Bei Ausbrüchen sollten Gesundheitsbehörden analytische epidemiologische Studien unter Verwendung von Datenquellen aus dem Herstellungs-, Bestell- oder Abrechnungsprozess in Erwägung zu ziehen, um die Abhängigkeit vom Erinnerungsvermögen befragter Studienteilnehmer zu verringern. Auch die Schnittstellen zwischen Lebensmittelrückverfolgung und -vorwärtsverfolgung einerseits und epidemiologischen Untersuchungen andererseits müssen methodisch weiterentwickelt werden.

2. Detaillierte Bevölkerungssurveys über Verzehrgewohnheiten, stratifiziert nach Alter, Geschlecht und Saisonalität, sollten in regelmäßigen $\mathrm{Ab}$ ständen durchgeführt werden, um bereits in der Phase der explorativen Erhebung die Hypothesengenerierung bei mutmaßlich lebensmittelbedingten Ausbrüchen zu unterstützen.

\section{Klinische Surveillance}

3. Die Ärzteschaft sollte intensiver motiviert und in die Verantwortung genommen werden, ihre Meldepflicht vollständig und ohne Zeitverzug wahrzunehmen. Hierzu gehört ein regelmäßiger kollegialer Austausch zwischen klinisch tätigen Ärzten und dem ÖGD sowie auch die ärztliche Aus- und fachärztliche Weiterbildung.

4. Das in Entwicklung befindliche System eines deutschlandweiten digitali- 
sierten Systems zur Erfassung von Infektionsdaten sollte zügig umgesetzt werden, um sein Potenzial entfalten zu können.

5. Über geeignete bestehende Regelwerke (Standesrecht, Abrechnungsmodalitäten o. a.) sollten Ärzte und Krankenhäuser motiviert und ggf. auch verpflichtet werden können, sich an Sentinel-Surveillance-Systemen zu beteiligen.

6. Die Mortalitätserfassung sollte so gestaltet werden, dass Landesbehörden und RKI mindestens wöchentlich die Anzahl der Todesfälle digital übermittelt bekommen.

\section{Molekulare Surveillance}

7. Anforderungen für Labordiagnostik, die Durchführung von Feintypisierung sowie der Probenversand zu entsprechenden Referenzlaboren sollten so geregelt werden, dass von den anfordernden Ärzten und den durchführenden Laboren die auch epidemiologisch notwendigen mikrobiologischen Untersuchungen zeitnah durchgeführt werden können. Mindestens für bestimmte Erreger (z. B. EHEC, Listerien, bestimmte Salmonellen-Serovare) sollte für Deutschland eine systematische, flächendeckende und zeitnahe Feintypisierung erfolgen [62].

\section{Erfassung der Versorgungslage}

8. Länder sollten Erfassungssysteme entwickeln, die jederzeit in der Lage sind, die Auslastung von Versorgungsstrukturen (z. B. spezieller Behandlungsplätze) überwachen zu können.

\section{Epidemiemanagement}

\section{Mobilisierung personeller Ressourcen}

9. Auf Ebene der Landesbehörden sollte in Deutschland ein Pool von mindestens 25 bis 30 Infektionsepidemiologen aufgebaut werden, um Gesundheitsämter beim Infektionsschutz, bei der Ausbrucherkennung und -untersuchung zu unterstützen. Die hierfür erforderliche Qualifizierung könnte das bestehende zweijährige Aus- bildungsprogramm für Angewandte Epidemiologie des RKI vermitteln, wenn es entsprechend finanziell unterstützt würde.

10. Im Falle eines geografisch begrenzten Geschehens sollte der ÖGD frühzeitiger von der Möglichkeit Gebrauch machen, Mitarbeiter im Sinne der Amtshilfe aus anderen thematischen Bereichen derselben Behörden oder aber aus Behörden in weniger betroffenen Regionen abzuordnen.

11. Die Vergütungsbedingungen insbesondere für ärztliches Personal im ÖGD sollten attraktiver gestaltet werden, um gegenüber dem klinisch-medizinischen Arbeitsmarkt wettbewerbsfähig zu werden.

12. Im öffentlichen Gesundheitsdienst sollten Qualifizierungsangebote im Bereich des Infektionsschutzes intensiviert werden.

\section{Koordination}

13. Ein in Berlin angesiedeltes, ressortübergreifendes Lagezentrum mit einem dauerhaft einsetzbaren Sekretariats- und Technikteam sollte vorgehalten werden, damit alle betroffenen Bundesbehörden dieses je nach Lage mit Fachexperten besetzen und nutzen können.

14. Bei Gefährdungslagen der öffentlichen Gesundheit von nationaler und internationaler Bedeutung (wie im Rahmen der internationalen Gesundheitsvorschriften definiert) sollte es den zuständigen Bundesbehörden ermöglicht werden, zusätzliche, eng definierte und zeitlich befristete Befugnisse zu erhalten, um z. B. epidemiologische Untersuchungen eigenständig betreiben oder personenbezogene Daten erheben zu können.

\section{Kommunikation}

\section{Abgestimmte Kommunikation}

15. Kommunal-, Landes- und Bundesbehörden sollten Informationen an die Öffentlichkeit, die auch von nationaler Bedeutung sein können, erst nach Abstimmung mit bzw. nach ausreichender Vorankündigung bei anderen betroffenen Behörden veröffentlichen.

16. Bundes- und Landesbehörden sollten in Zeiten außerhalb akuter Geschehen ihre Arbeitsweisen und Methoden für Ausbruchuntersuchungen kontinuierlich vermitteln bzw. im Ereignisfall entsprechendes Informationsmaterial verfügbar haben, sodass die Öffentlichkeit diese nachvollziehen kann.

\section{Wissenschaftlicher Diskurs}

17. Wissenschaftliche Fachgesellschaften und andere Nicht-Regierungsorganisationen im Bereich des Gesundheitswesens sollten einschlägige Empfehlungen zur guten wissenschaftlichen Praxis um den Aspekt einer fachlich fundierten und verantwortungsvollen Öffentlichkeitsarbeit ergänzen und umsetzen.

\section{Schlussfolgerung}

Die hier diskutierten Probleme der Ausbruchbewältigung zeigen, dass durchaus ein erheblicher Verbesserungsbedarf in Bereichen der Ausbrucherkennung, Ausbruchuntersuchung und Kommunikation besteht. Während einige dieser Aspekte bislang kaum Beachtung fanden (Labordiagnostik), wurde die öffentliche Diskussion mehrheitlich von Themenbereichen geprägt, die bei näherer Betrachtung entweder nicht haltbar (z. B. Ermittlungsfehler) oder aber im Vergleich zu den hier diskutierten Problemfeldern in ihrer Bedeutung nachrangig sind (z. B. Zeitverzug durch Übermittlung vom Gesundheitsamt an das RKI).

Die hier aufgeführten Empfehlungen erheben keinesfalls den Anspruch auf Vollständigkeit und wurden teilweise bereits bei der Aufarbeitung der H1N1Influenzapandemie identifiziert. Während einige Aspekte, wie z. B. die Verkürzung der Übermittlungsfristen, bereits im Rahmen geplanter Gesetzesänderungen angegangen wurden, sollten auch Maßnahmen nicht vernachlässigt werden, deren Umsetzung erhebliche öffentliche Mittel für den ÖGD erfordern würden (z. B. Personalressourcen). 
Der HUS-Ausbruch hat gezeigt, dass solche Ereignisse unvorhersehbar und unvermeidbar jederzeit auftreten und immense wirtschaftliche Kosten erzeugen können, die die erforderlichen Investitionen in den Bereich der Früherkennung und Eindämmung von Ausbrüchen bei Weitem übersteigen. Diese gesellschaftliche Gesamtbilanz sollte trotz knapper Ressourcen im öffentlichen Gesundheitsdienst berücksichtigt werden.

\section{Korrespondenzadresse}

\section{Prof. Dr. G. Krause}

Robert Koch-Institut

DGZ-Ring 1, 13086 Berlin

KrauseG@rki.de

Danksagung. Dank gilt Doris Altmann für Datenbankabfragen sowie Jessica Bielecke und Inge Mücke für die Literaturpflege. Wir danken auch Helen Bernard, Susanne Glasmacher, Bettina Rosner und Dirk Werber für kritische Kommentierungen sowie den Mitarbeiterinnen und Mitarbeitern der Abteilung Infektionsepidemiologie am RKI für zahlreiche Dokumentationen und Veröffentlichungen, auf die dieses Manuskript zurückgreifen konnte. Vor allem jedoch möchten wir an dieser Stelle allen Kolleginnen und Kollegen innerhalb und außerhalb des Institutes danken, die trotz schwieriger Rahmenbedingungen mit außerordentlichem Engagement zur Aufklärung dieses dramatischen Ausbruchs beitrugen.

Interessenkonflikt. Der korrespondierende Autor gibt für sich und seine Koautoren an, dass kein Interessenkonflikt besteht. Alle Autoren waren als Beschäftigte des RKI persönlich an der Bewältigung des Ausbruchs beteiligt.

\section{Literatur}

1. Communities Commission of the European. Lessons learned from the 2011 outbreak of Shiga toxin-producing Escherichia coli (STEC) 0104:H4 in sprouted seeds, SANCO/13004/2011, to be published in August 2012, 2012 (Zugegriffen: 29.11.2012)

2. RKI (2011) Abschließende Darstellung und Bewertung der epidemiologischen Erkenntnisse im EHEC 0104:H4 Ausbruch Deutschland 2011. (Zugegriffen: 29.11.2012)

3. Askar M, Faber MS, Frank C et al (2011) Update on the ongoing outbreak of haemolytic uraemic syndrome due to Shiga toxin-producing Escherichia coli (STEC) serotype 0104, Germany, May 2011. Euro Surveill 16(22):pii:19883

4. Frank C, Werber D, Cramer JP et al (2011) Epidemic profile of Shiga-toxin-producing Escherichia coli 0104:H4 outbreak in Germany. N Engl J Med 365(19):1771-1780

5. Frank C, Faber MS, Askar M et al (2011) Large and ongoing outbreak of haemolytic uraemic syndrome, Germany, May 2011. Euro Surveill 16(21):pii: 19878
6. RKI (2012) Infektionsepidemiologisches Jahrbuch meldepflichtiger Krankheiten für 2011. Robert Koch-Institut, Berlin

7. Rosner B, Bernard H, Werber D et al (2011) Epidemiologie des EHEC 0104:H4/HUS-Ausbruchs in Deutschland, Mai bis Juli 2011. JVerbr Lebensm 6:473-481

8. Buchholz U, Bernard H, Werber D et al (2011) German outbreak of Escherichia coli 0104:H4 associated with sprouts. N Engl J Med 365(19):17631770

9. Muller L, Kjelso C, Nielsen EM et al (2011) Danish outbreak investigation related to a large outbreak of Shiga toxin-producing E. coli o104 in Germany, May-June 2011- the important role of outlier investigation. ESCAIDE, Stockholm

10. Ivarsson S, Jernberg C, Bjorkholm B et al (2011) Rapid detection and communication of Swedish cases provided early puzzle pieces in the German STeC 0104 outbreak. ESCAIDE, Stockholm

11. Scharlach M, Diercke M, Dreesman J et al (2011) Outbreak of Shiga toxin-producing Escherichia coli serotype 0104:H4 in Germany: cohort study of a cluster in Lower Saxony (Poster). ESCAIDE, Stockholm

12. Krause G (2012) Outbreak of STEC O104:H4 associated with fenugreek sprout seeds - epidemic profile and investigation in Germany, 2011. Conference for emerging infectious diseases, Atlanta, 12.03.2012

13. Werber D, Krause G, Frank C et al (2012) Outbreaks of virulent diarrhoeagenic Escherichia coli - are we in control? BMC Med 10(1):11

14. European Food Safety Authority (EFSA) (2011) Tracing seeds, in particular fenugreek (Trigonelle foenum-graecum) seeds, in relation to the Shiga toxin-producing E. coli (STEC) 0104:H4 2011 Outbreaks in Germany and France. http://www.efsa.europa.eu/de/supporting/doc/176e.pdf (Zugegriffen: 29.11.2012)

15. Die Task Force EHEC, Bernard $\mathrm{H}$, Bisping $M$ et al (2011) Ergebnisbericht der Task Force EHEC zur Aufklärung des EHEC 0104:H4 Krankheitsausbruchs in Deutschland. J Verbr Lebensm 6(4):483495

16. King LA, Nogareda F, Weill FX et al (2012) Outbreak of shiga Toxin-producing escherichia coli 0104:H4 associated with organic fenugreek sprouts, France, June 2011. Clin Infect Dis 54(11):1588-1594

17. Pritchard L, Holden NJ, Bielaszewska M et al (2012) Alignment-free design of highly discriminatory diagnostic primer sets for escherichia coli 0104:H4 Outbreak Strains. PloS one 7(3):e34498

18. Mellmann A, Harmsen D, Cummings $C A$ et al (2011) Prospective genomic characterization of the German enterohemorrhagic Escherichia coli 0104:H4 outbreak by rapid next generation sequencing technology. PLoS One 6(7):e22751

19. Bielaszewska M, Mellmann A, Zhang W et al (2011) Characterisation of the Escherichia coli strain associated with an outbreak of haemolytic uraemic syndrome in Germany, 2011: a microbiological study. Lancet Infect Dis 11(9):671-676

20. Aurass $P$, Prager $R$, Flieger A (2011) EHEC/EAEC 0104:H4 strain linked with the 2011 German outbreak of haemolytic uremic syndrome enters into the viable but non-culturable state in response to various stresses and resuscitates upon stress relief. Environ Microbiol 13(12):3139-3148
21. Hamburg-Eppendorf Universitätsklinikum (2011) Pressemitteilung vom 7. Juni 2011: UKE hat Notfallversorgung wieder in vollem Umfang aufgenommen. http://www.uke.de/medien/index_74852.php (Zugegriffen: 29.11.2012)

22. Bild-Zeitung (2011) Schon 18 EHEC-Tote! Hamburgs Bürgermeister Scholz ruft zu Blutspenden auf 2011. http://www.bild.de/news/inland/ehec/ buergermeister-scholz-spendet-blut-18192818. bild.html (Zugegriffen: 29.11.2012)

23. Hänel C-M, Jüptner C, Lorenz K et al (2011) Chronologie des EHEC 0104:H4 Ausbruchsgeschehens bis zur Gründung der länderübergreifenden Task Force EHEC aus Sicht der Lebensmittelüberwachung in Schleswig-Holstein am Beispiel des Kreises Schleswig-Flensburg. J Verbr Lebensm 6(6):497-501

24. Siegmund-Schultze N, Rieser S (2012) Neue Meldepflichten:Verzögerung wegen Kostenstreits. Dtsch Ärztebl 109:A564.4

25. Lohmann M, Epp A, Röder B, Böl GF (2013) Risikokommunikation von Bundesinstitutionen bei Ausbruchsgeschehen. Bundesgesundheitsbl Gesundheitsforsch Gesundheitsschutz 56:102-109

26. Sandman PM, Lanard J (2011) Explaining and proclaiming uncertainty: risk communication lessons from Germany's deadly E. coli outbreak. http://www.psandman.com/col/GermanEcoli.htm (Zugegriffen: 29.11.2012)

27. Deutsche Gesellschaft für Infektiologie. EHEC und Antibiotikabehandlung, 2011. http://www. dgi-net.de/images/stories/DGI-Positionspapier_ EHEC_und_Antibiotika_20110601.pdf (Zugegriffen: 29.11.2012)

28. Deutsche Gesellschaft für Nephrologie. EHEC Informationen für das Fachpublikum, 2011. http:// www.dgfn.eu/aktuell/ehec-informationen/fuerdas-fachpublikum/ehec-und-antibiotikabehandlung.html (Zugegriffen: 29.11.2012)

29. Deutsche Gesellschaft für Allgemeinmedizin und Familienmedizin. EHEC-Ansteckung:„Gefährlichster Erreger heißt ... Panik". http://www.degam. de/index.php?id=ehec-ansteckunggefhrlichste (Zugegriffen: 01.06. 2011 um 12:48, inzwischen nicht mehr abrufbar, Ausdruck liegt dem Autor vor)

30. ARD Wochenspiegel - Hygiene-Experte Dr. KlausDieter Zastrow zur Ausbreitung des EHEC-Erregers, 05.06.2011

31. Lehn B vom (2011) „Katastrophaler Unsinn“. Berliner Zeitung 21.06.2011

32. (o A) (2011) Killer-Keime könnten gezüchtet worden sein. Bild.de 03.06.2011

33. Kekulé AS (2011) EHEC-Ausbruch gibt Rästel auf auch unheimliche. Tagesspiegel 25.05.2011

34. Kekulé AS (2011) Ausbreitung des EHEC-Darmkeims ist "großes Rätsel", 23.05.2011

35. (o A) (2011) ARD Morgenmagazin - Interview mit Prof. Dr. Alexander Kekulé zu aktuellen EHEC-Erkenntnissen: ARD, 06.06.2011

36. (o A) (2011) Wie merke ich, dass ich infiziert bin? Berliner Kurier 24.05.2011

37. Lossau N (2011) „EHEC kann auch das Gehirn angreifen“. Welt online 03.06.2011

38. WHO. Foodborne disease outbreaks: guidelines for investigation and control 2008. http://apps.who.int/iris/bitstream/10665/43771/1/9789241547222_eng.pdf (Zugegriffen: 29.11.2012)

39. Barton Behravesh Casey, Mody Rajal K, Jungk J et al (2011) 2008 Outbreak of salmonella saintpaul infections associated with raw produce. $\mathrm{N}$ Engl $\mathrm{J}$ Med 918-927 


\section{Leitthema}

40. Wilking H, Gotsch U, Meier H et al (2012) Identifying risk factors for shiga toxin-producing Escherichia coli by payment information. Emerg Infect Dis 18(1):169-170

41. Harendza S (2011) „HUS diary“ of a German nephrologist during the current EHEC outbreak in Europe. Kidney Int 80(7):687-689

42. Rieser S (2011) Ausbreitung bedrohlicher Erkrankungen: Behörden sollen rascher kooperieren. Dtsch Arztebl 108(36):A-1818

43. Anonymous (2011) Contaminated food for thought. Nature 474:251

44. Krause G, Benzler J, Reiprich G, Gorgen R (2006) Improvement of a national public health surveillance system through use of a quality circle. Euro Surveill 11(11):246-248

45. Werber D, Behnke SC, Fruth A et al (2007) Shiga Toxin-producing escherichia coli infection in Germany- different risk factors for different age groups. Am J Epidemiol 165(4):425-434

46. Köpke K (2009) Influenza-Überwachung: Surveillance der Kranheitslast. Dtsch Arztebl 106(5):176

47. Buda S, Haas W (2011) Arbeitsgemeinschaft Influenza: Um Unterstützung wird gebeten. Dtsch Arztebl 108(4):150

48. Robert Koch-Institut (2011) Influenzasurveillance in der ersten postpandemischen Saison. Epid Bull 149

49. Swaminathan B, Gerner-Smidt P, Ng LK et al (2006) Building PulseNet International: an interconnected system of laboratory networks to facilitate timely public health recognition and response to foodborne disease outbreaks and emerging foodborne diseases. Foodborne Pathog Dis 3(1):36-50

50. 128. Bundesgesetz zur Überwachung von Zoonosen und Zoonoseeregern (Zoonosengesetz). Bundesgesetzblatt für die Republik Österreich, 2005. http://www.ris.bka.gv.at/Dokument. $w x e$ ?Abfrage $=$ BgblAuth\&Dokumentnummer=BGBLA_2005_I_128 (Zugegriffen: 29.11.2012)

51. Krause G, Gilsdorf A, Becker J et al (2010) Erster Erfahrungsaustausch zur H1N1-Pandemie in Deutschland 2009/2010. Bundesgesundheitsbl Gesundheitsforsch Gesundheitsschutz 53(5):510519

52. Taylor E, Kastner J, Renter D (2010) Challenges involved in the salmonella saintpaul outbreak and lessons learned. J Public Health Manag Pract 16(3):221-231

53. Gerst T (2011) Interview mit Dr. med. Ute TeichertBarthel, Vorsitzende des Bundesverbandes der Ärztinnen und Ärzte des Öffentlichen Gesundheitsdienstes (BVÖGD):,"Wir haben die Chance, etwas zu bewegen". Dtsch Ärztebl 108(45):A-2393

54. Krause G, Stefanoff P, Moren A (2009) Applied epidemiology training in Europe: quite a success - but more to be done. Euro Surveill 14(43): $\mathrm{pii}=19375$

55. Epidemiologists Council of State and Territorial. 2006 National assessment of epidemiologic capacity: findings and recommendations [cited 2007 Jun 21]. http://www.cste.org/pdffiles/2007/ 2006CSTEECAFINALFullDocument.pdf

56. Schweizerische Eidgenossenschaft. Revision Epidemiengesetz EpG 2012. http://www.bag.admin. ch/themen/medizin/03030/03209/03210/index. html?lang=de (Zugegriffen: 29.11.2012)

57. Die Bundesversammlung, das Schweizer Parlament. Epidemiegesetz Revision. $2012 \mathrm{http} / /$ www.parlament.ch/d/suche/seiten/geschaefte.aspx?gesch_id=20100107 (Zugegriffen: 29.11.2012)
58. Code of Federal Regulations - Title 42: Public Health. 1. Oktober 2010. http://cfr.vlex.com/vid/ 70-measures-event-inadequate-local-19796469 (Zugegriffen: 29.11.2012)

59. (o A) (2012) Foodwatch. Im Bockshorn - Die EHECKrise im Frühsommer 2011. http://www.bag.admin.ch/themen/medizin/03030/03209/03210/index.html?lang=de (Zugegriffen: 29.11.2012)

60. Rossmann C, Brosius HB (2013) Die Risiken der Risikokommunikation - Die Rolle der Massenmedien in der Risikokommunikation. Bundesgesundheitsbl Gesundheitsforsch Gesundheitsschutz 56:118-123

61. Glasmacher S (2012) Sind wir gefährdet? Krisenkommunikation für ein Bundesinstitut auf dem Gebiet des Gesundheitsschutzes. In: Dernbach B, Kleinert C, Münder H (Hrsg) Handbuch Wissenschaftskommunikation. Springer, Berlin Heidelberg New York Tokyo, S 303-318

62. Werber D, Stark K, Flieger Antje, Tietze E (2013) Zur Rolle der Feintypisierung in der Ausbruchserkennung. Bundesgesundheitsbl Gesundheitsforsch Gesundheitsschutz 56:38-41

\section{Hier steht eine Anzeige.}

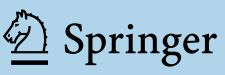

